\title{
Extracorporeal membrane oxygenation as a bridge to lung transplantation in the United States: An evolving strategy in the management of rapidly advancing pulmonary disease
}

\author{
Awori J. Hayanga, MD, MPH, ${ }^{a}$ Jonathan Aboagye, MD, MPH, ${ }^{\mathrm{b}}$ Stephen Esper, MD, MBA, ${ }^{c}$ \\ Norihisa Shigemura, MD, PhD, ${ }^{\mathrm{d}}$ Christian A. Bermudez, MD, ${ }^{\mathrm{d}}$ Jonathan D'Cunha, MD, PhD, ${ }^{\mathrm{d}}$ and \\ Jay K. Bhama, MD
}

\begin{abstract}
Objective: Improvements in technology have led to a resurgence in the use of extracorporeal membrane oxygenation as a bridge to lung transplantation. By using a national registry, we sought to evaluate how short-term survival has evolved using this strategy.
\end{abstract}

\begin{abstract}
Methods: With the use of the United Network for Organ Sharing database, we analyzed data from 12,458 adults who underwent lung transplantation between 2000 and 2011. Patients were categorized into 2 cohorts: 119 patients who were bridged to transplantation using extracorporeal membrane oxygenation and 12,339 patients who were not. The study period was divided into four 3-year intervals: 2000 to 2002, 2003 to 2005, 2006 to 2008, and 2009 to 2011. With Kaplan-Meier analysis, 1-year survival was compared for the 2 cohorts of patients in each of the time periods. A propensity score-adjusted Cox regression model was used to estimate the risk of 1-year mortality.

Results: Of the total number of recipients, 4 (3.4\%) were bridged between 2000 and 2002, $17(14.3 \%)$ were bridged between 2003 and 2005, 31 (26.1\%) were bridged between 2006 and 2008, and 67 were bridged $(56.3 \%)$ between 2009 and 2011. Recipients bridged using extracorporeal membrane oxygenation were more likely to be younger and diabetic and to have higher serum creatinine and bilirubin levels. The 1-year survival for those bridged with extracorporeal membrane oxygenation was significantly lower in subsequent periods: $25.0 \%$ versus $81.0 \%$ (2000-2002), $47.1 \%$ versus $84.2 \%$ (2006-2008), and $74.4 \%$ versus $85.7 \%$ (20092011). However, this survival progressively increased with each period, as did the number of patients bridged using extracorporeal membrane oxygenation.
\end{abstract}

Conclusions: Short-term survival with the use of extracorporeal membrane oxygenation as a bridge to lung transplantation has significantly improved over the past few years. (J Thorac Cardiovasc Surg 2015;149:291-6)

Lung recipients are prioritized for transplantation on the basis of clinical severity. Nevertheless, many deteriorate on the waiting list and become dependent on ventilator support. There is a growing number of high-acuity patients whose disease progresses beyond conventional ventilation or standard pharmacologic support. This has prompted the use of

\footnotetext{
From the DeVos Heart and Lung Transplantation Program Spectrum Health, ${ }^{a}$ Michigan State University, Grand Rapids, Mich; Johns Hopkins School of Public Health, ${ }^{\mathrm{b}}$ Baltimore, Md; Department of Anesthesiology, ${ }^{\mathrm{c}}$ University of Pittsburgh Medical Center, Pittsburgh, Pa; Department of Cardiothoracic Surgery, ${ }^{\mathrm{d}}$ University of Pittsburgh Medical Center, Pittsburgh, Pa; and Heart and Vascular Center, ${ }^{\text {e }}$ Department of Cardiothoracic Surgery, University of Iowa Hospitals and Clinics, Iowa City, Iowa.

Disclosures: Authors have nothing to disclose with regard to commercial support. Read at the 94th Annual Meeting of The American Association for Thoracic Surgery, Toronto, Ontario, Canada, April 26-30, 2014.

Received for publication April 10, 2014; revisions received Aug 13, 2014; accepted for publication Aug 20, 2014.

Address for reprints: Jay K. Bhama, MD, Heart and Vascular Center, Department of Cardiothoracic Surgery, University of Iowa Hospitals and Clinics, 200 Hawkins Dr, Iowa City, IA 52242 (E-mail: jay-bhama@uiowa.edu). $0022-5223 / \$ 36.00$

Copyright (c) 2015 by The American Association for Thoracic Surgery http://dx.doi.org/10.1016/j.jtcvs.2014.08.072
}

mechanical support to serve as a "bridge" until a suitable donor becomes available.

Two decades ago, however, mechanical support was largely considered a contraindication to lung transplantation in view of the poor outcomes achieved with its early use. This morbidity was related to hemolysis, infection, bleeding, and hemodynamic instability, among many other issues, each of which fueled a growing reluctance to broad endorsement. ${ }^{1-10}$ However, improved results in the use of extracorporeal membrane oxygenation (ECMO) as a rescue therapy for primary graft dysfunction after lung transplantation has resulted in a resurgence of interest in its use. ${ }^{11-15}$

Recent clinical advances have served to further increase the efficacy and safety of contemporary ECMO use as a whole. ${ }^{7,16}$ Many centers have published improved results with ECMO, but, even collectively, these have been based on small numbers. ${ }^{7,16-19}$ Furthermore, the reports have had mixed conclusions resulting in equivocation and a general lack of consensus. ${ }^{3,4,7,8,20-24}$ Nevertheless, the growing trend in recent series is invariably favorable, and supportive evidence in our own practice prompted this 


$$
\begin{aligned}
& \text { Abbreviations and Acronyms } \\
& \text { CI = confidence interval } \\
& \text { ECMO }=\text { extracorporeal membrane oxygenation } \\
& \text { HR }=\text { hazard ratio } \\
& \text { LAS }=\text { lung allocation score } \\
& \text { OR } \quad \text { odds ratio }
\end{aligned}
$$

national analysis. ${ }^{19}$ We sought to evaluate the differences in short-term survival with the use of ECMO as a bridge to lung transplantation, with a focus on temporal trends over the past 12 years to establish whether an objective improvement in survival exists that would justify the utility of this practice.

\section{MATERIALS AND METHODS \\ Study Design}

We performed a retrospective evaluation of data obtained from Scientific Registry of Transplant Recipients Standard Transplant Analysis and Research files provided by the United Network for Organ Sharing. This comprised a data set of a prospectively collected open cohort of consecutive US adult lung transplant recipients who underwent transplantation between January 2000 and December 2011. Both patient-level data and transplantation center data were provided in a de-identified format. We excluded patients with missing information on pertinent variables.

The cohort was subdivided into 2 groups, comprising those in whom ECMO support had been used as a bridge to lung transplantation and those in whom it had not. To analyze trends in outcome for the 2 groups, the study period was stratified into four 3-yearly intervals, comprising 2000 to 2002 , 2003 to 2005,2006 to 2008 , and 2009 to 2011.

\section{Statistical Analysis}

A total of 15,067 lung transplant recipients were identified in the study period. After excluding patients with missing information on pertinent variables, 12,458 were included in the analysis. Of this total, 119 recipients $(1.0 \%)$ were bridged with ECMO and 12,339 recipients $(99.0 \%)$ were not. We compared baseline characteristics between the 2 groups. Student $t$ test and Wilcoxon rank-sum test were used to compare normally and skewed continuous variables, respectively. A Fisher exact test was used to compare categoric variables. Survival analysis was performed using a Kaplan-Meier method to estimate 1-year survival, comparing the 2 cohorts of patients within each time interval with a log-rank test.

By using multivariate logistic regression, we estimated propensity scores for the likelihood of being supported with ECMO. All variables in Table 1 were used in estimating the propensity scores. A Cox regression adjustment was performed on the propensity scores to estimate the risk of 1-year mortality after lung transplantation between patients who were bridged with ECMO and those who were not. Patients with missing data were excluded from the model using case-wise deletion. A Cox model with a stepwise regression selection method with $P=.2$ was used to identify the predictors of 1 -year mortality among patients who had ECMO as a bridge to lung transplantation using the recipient, donor, and transplant-related variables listed in Table 1. We estimated the trends in the risk of dialysis-dependent renal failure after transplantation using propensity score-adjusted logistic regression. Patients who had ever been dialyzed before transplantation were excluded from this analysis.

Mean values and medians are displayed with their standard deviations and interquartile ranges. Hazard ratios (HRs) are presented with their
95\% confidence intervals (CIs). All the analyses were carried out with Stata 12.0 (StataCorp LP, College Station, Tex).

\section{RESULTS \\ Cohort Characteristics}

Four patients $(3.4 \%)$ were bridged with ECMO from 2000 to 2002,17 patients $(14.3 \%)$ were bridged with ECMO from 2003 to 2005,31 patients $(26.1 \%)$ were bridged with ECMO from 2006 to 2008, and 67 patients $(56.3 \%)$ were bridged with ECMO from 2009 to 2011. Patients bridged on ECMO were more likely to be younger and diabetic and to have higher serum creatinine and bilirubin levels. The 2 groups also differed by distribution of thoracic diagnoses, and patients bridged with ECMO had longer graft ischemic times and shorter wait-list duration (Table 1).

\section{Primary Outcome}

The 1-year survival in patients bridged with ECMO was significantly shorter than in those within the non-ECMO group in the time periods 2000 to $2002(25.0 \%$ vs $81.0 \%), 2006$ to $2008(47.1 \%$ vs $84.2 \%)$, and 2009 to 2011 (74.4\% vs $85.7 \%)$. However, the 2 groups did not differ significantly from each other between 2003 and 2005 (76.5\% vs $84.5 \%$ ) (Figure $1, A-D)$. In the propensity score-adjusted analysis, patients bridged with ECMO had a significantly higher risk of 1-year mortality after transplantation in the periods 2000 to 2002 (HR, 7.15; 95\% CI, 2.23-22.89; $P=.001$ ), 2006 to 2008 (HR, 6.24; 95\% CI, 3.77-10.33; $P<.001$ ), and 2009 to 2011 (HR, 1.96; $95 \%$ CI, 1.20-3.21; $P=.007)$. However, no difference in 1-year mortality after transplantation was observed for the 2 groups from 2003 to 2005 (HR, 1.62; 95\% CI, $0.61-4.350 ; P=.34$ ) (Table 2). Figure 2 depicts the trends in the risk of 1-year mortality for the ECMO group and non-ECMO group.

\section{Predictors of Mortality}

Predictors of 1-year mortality in patients who had been bridged using ECMO support included recipient age more than 35 years (HR, $4.69 ; 95 \% \mathrm{CI}, 1.33-16.46 ; P=.02)$, a diagnosis of cystic fibrosis (HR, 5.38; 95\% CI, 1.3920.87; $P=.03$ ), and patients categorized in the "other lung diagnosis" group (HR, 2.49; 95\% CI, 1.21-5.15; $P=.01)$ (Table 3).

\section{Secondary Outcome}

We observed that patients who were bridged with ECMO had a higher risk of dialysis-dependent renal failure for the periods 2003 to 2005 (odds ratio [OR], 11.1; 95\% CI, 2.40-51.40; $P=.002$ ), 2006 to 2008 (OR, 13.57; 95\% CI, 6.44-28.6; $P<.001$ ), and 2009 to 2011 (OR, 3.31; 95\% CI, 1.66-6.58; $P=.007$ ). From 2000 to 2002 , the 2 groups had a similar risk of dialysis- 
TABLE 1. Comparison of characteristics of lung transplant recipients supported and not supported with extracorporeal membrane oxygenation bridge

\begin{tabular}{|c|c|c|c|}
\hline Variables & $\begin{array}{c}\text { ECMO } \\
(\mathbf{N}=119)\end{array}$ & $\begin{array}{l}\text { Non-ECMO } \\
(\mathrm{N}=\mathbf{1 2 , 3 3 9 )} \\
\end{array}$ & $P$ value \\
\hline \multicolumn{4}{|l|}{ Recipient-related } \\
\hline Median age, y (IQR) & $51(34-60)$ & $57(48-63)$ & $<.001$ \\
\hline Male gender, $\mathrm{n}(\%)$ & $74(62.2)$ & $7007(56.8)$ & .27 \\
\hline White, n (\%) & $98(82.4)$ & $10,482(85.0)$ & .12 \\
\hline \multicolumn{4}{|l|}{ Recipient BMI $\left(\mathrm{kg} / \mathrm{m}^{2}\right), \mathrm{n}(\%)$} \\
\hline Normal (18.5-24.9) & $45(37.8)$ & $5097(41.3)$ & .33 \\
\hline Underweight $(<18.9)$ & $1215(9.9)$ & $14(11.8)$ & .33 \\
\hline Overweight (25-29.9) & $4271(34.6)$ & $37(31.1)$ & .33 \\
\hline Obese $(>30)$ & $1756(14.2)$ & $23(19.3)$ & .33 \\
\hline $\begin{array}{l}\text { Median creatinine, } \mathrm{mg} / \mathrm{dL} \\
\text { (IQR) }\end{array}$ & $0.8(0.6-0.9)$ & $0.8(0.7-1.0)$ & .002 \\
\hline $\begin{array}{l}\text { Median total bilirubin, } \\
\mathrm{mg} / \mathrm{dL} \text { (IQR) }\end{array}$ & $0.7(0.4-1.2)$ & $0.5(0.3-0.7)$ & $<.001$ \\
\hline Diabetes, n (\%) & $28(23.5)$ & $1903(15.4)$ & .02 \\
\hline \multicolumn{4}{|l|}{ Thoracic diagnosis } \\
\hline IPF, n (\%) & $48(40.3)$ & $3886(31.5)$ & $<.001$ \\
\hline COPD/emphysema, n (\%) & $18(15.1)$ & $3993(32.4)$ & $<.001$ \\
\hline Cystic fibrosis, n (\%) & $20(16.8)$ & $1557(12.6)$ & $<.001$ \\
\hline Other, n $(\%)$ & $33(27.7)$ & $2903(23.5)$ & $<.001$ \\
\hline \multicolumn{4}{|l|}{ Donor- and transplant-related } \\
\hline Donor smoking, n (\%) & $17(14.3)$ & 1874 & .90 \\
\hline Median donor age, y (IQR) & $30(21-43)$ & $31(21-45)$ & .65 \\
\hline Mean ischemic time, $\mathrm{h}(\mathrm{SD})$ & $5.9(1.8)$ & $5.0(1.7)$ & $<.001$ \\
\hline $\begin{array}{l}\text { Median wait-list duration, } \\
\text { d (IQR) }\end{array}$ & $23(7-117)$ & $111(31-321)$ & $<.001$ \\
\hline
\end{tabular}

$\overline{B M I}$, Body mass index; $C O P D$, chronic obstructive pulmonary disease; $E C M O$, extracorporeal membrane oxygenation; $I P F$, idiopathic pulmonary fibrosis; $I Q R$, interquartile range; $S D$, standard deviation.

dependent renal failure (OR, 3.32; 95\% CI, 0.32-34.00; $P=.31)$ (Table 4).

\section{DISCUSSION}

In this study, we evaluate longitudinal trends in shortterm survival with the use of ECMO and report significant increases in volume and improvements in short-term survival in the recent past. A growing number of transplant centers continue to report favorable outcomes, but often in the past, there have been conflicting results from single-center studies underpowered to resolve the conflict. $^{3,8,9}$ This report, currently the largest series in the literature made possible through the use of a national data registry, allows a more robust denominator to better answer the question at hand. A time series analysis allowed a more granular evaluation of the incremental gains made over the past decade. In this vein, we report increases in 1-year survival from $25 \%$ in the earliest period between 2000 and 2002 to $75 \%$ between 2009 and 2011. This improvement in contemporary survival is consistent with reports by Hämmäinen and colleagues ${ }^{3}$ in
2 separate studies by our own group, each of which reported individual survival of $75 \%, 74 \%$, and $74 \%$, respectively. ${ }^{3,19,25}$ We believe that the basis for this improvement in survival is likely multifactorial.

We demonstrated a decreased risk of dialysis-dependent renal failure with time. This was the only common surgical complication which has data consistently available to allow meaningful analysis. We deem these improvements to be a multifactorial phenomenon, likely also associated with the introduction of the lung allocation score (LAS) in 2005, a plausible contributor to the shorter wait-list times observed over the years. We hypothesize that as the LAS system is further reanalyzed and revised, data presented in such analyses may urge the incorporation of ECMO as one of the variables in the calculation.

There is a growing institutional experience nationwide with the use of extracorporeal support, and more transplant centers are opting to use ECMO as a bridge. These gains are, in part, associated with improvements in technologic design in the circuitry used for extracorporeal support that has occurred in the last many years. A major improvement has been the development of hollow-fiber, polymethylpentine oxygenators (ie, Quadrox, MAQUET, Hirrlingen, Germany), which decreased the incidence of thrombosis and plasma leak. The use of hollow fiber membrane oxygenators as opposed to solid silicone membrane oxygenators allow for a reduced amount of heparin administration with a comparatively shorter activated clotting time and improved oxygenation. ${ }^{3,11,12,26}$ Another major advantage was the introduction of the dual-lumen Avalon (MAQUET) cannula that allows for single-site cannulation for venovenous ECMO and potentially improved mobilization and even ambulation in the preoperative period. Another formidable advance has been the development of more sophisticated levitated centrifugal pumps (eg, CentriMag [Thoratec Corp, Pleasanton, Calif], Rotaflow [MAQUET], Revolution [Sorin Group USA, Arvada, Colo], and Cardiohelp [MAQUET]) and heparin-bonded circuits that have allowed for less blood element damage (ie, hemolysis), lower inflammation, and lesser need for anticoagulation. These factors have each provided incremental improvements and minimized complications related to instrument failure, complement activation, and hemolysis, allowing circuits to last longer and cost less. ${ }^{3}$

In addition, we further postulate that patient and donor selection play a role in the increase in survival with an observed shift in emphasis toward younger patients with single organ disease. ${ }^{19}$ Simultaneously, there has been increased use of dual-lumen single cannula technology (ie, Avalon), likely resulting in lowering of sedation requirements, encouraging mobilization and further minimizing the morbidity associated with protracted immobilization and prolonged mechanical ventilation. ${ }^{19}$ The growing trend toward double as opposed to single lung 

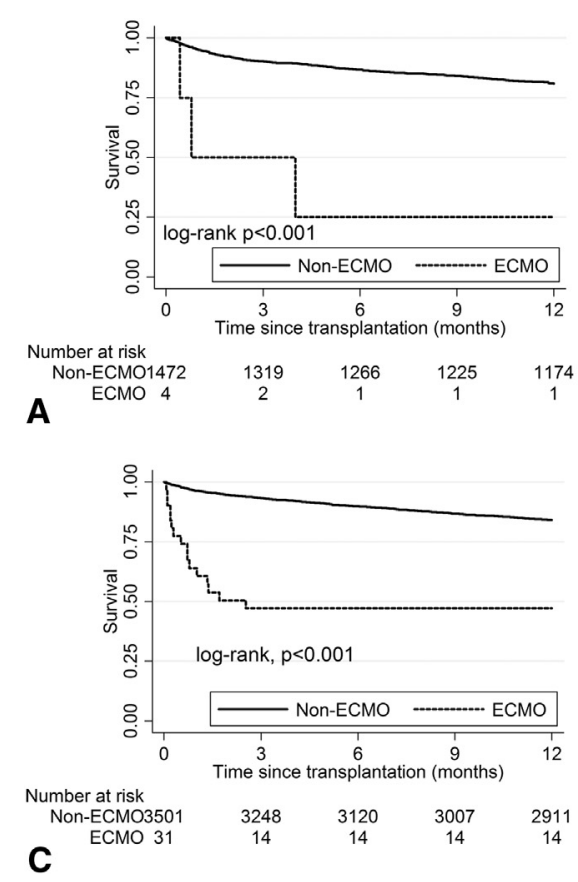
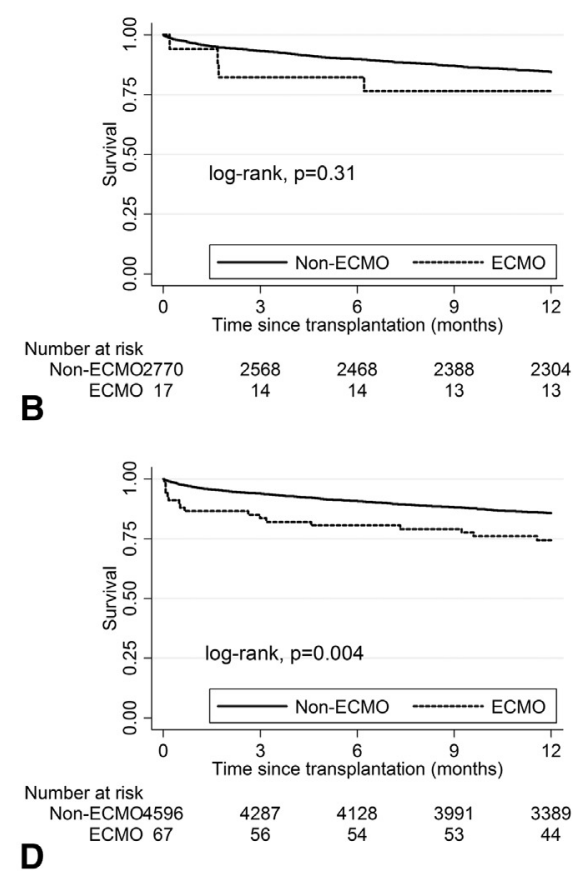

FIGURE 1. Kaplan-Meier curves comparing survival between patients with and without ECMO bridge to lung transplantation according to time interval: 2000 to 2002 (A), 2003 to 2005 (B), 2006 to 2008 (C), and 2009 to 2011 (D). ECMO, Extracorporeal membrane oxygenation.

transplantation in this population likely has been associated with improved outcomes and is potentially a strong driver of improved survival overall as the number of double lung transplantations continues to increase countrywide. $^{12}$ This finding is consistent with those from high-volume centers with a documented experience in extracorporeal support. ${ }^{25}$ These factors support the reports that double lung transplantation is likely the intervention of choice for patients bridged using ECMO, a notion previously suspected but largely unproven because of the lack of sufficient power in other studies that have attempted to answer this question. ${ }^{8,25,27-29}$

\section{Study Limitations}

First, the lack of granular detail makes it impossible to distinguish between venoarterial and venovenous ECMO. However, the type of ECMO has not been demonstrated to exert an effect on outcomes. ${ }^{2,19}$ Second, we evaluate

TABLE 2. Propensity score-adjusted 1-year risk of death after lung transplantation comparing extracorporeal membrane oxygenation with nonextracorporeal membrane oxygenation cases stratified into four 3-year intervals

\begin{tabular}{clc}
\hline Period & HR $(\mathbf{9 5} \%$ CI $)$ & $\boldsymbol{P}$ value \\
\hline $2000-2002$ & $7.15(2.23-22.89)$ & .001 \\
$2003-2005$ & $1.62(0.61-4.35)$ & .34 \\
$2006-2008$ & $6.24(3.77-10.33)$ & $<.001$ \\
$2009-2011$ & $1.96(1.20-3.21)$ & .007 \\
\hline
\end{tabular}

$C I$, Confidence interval; $H R$, hazard ratio. only short-term outcomes in this report, and the long-term outcomes, as they become apparent in the near future, will require evaluation over the next few years. Third, the cumulative experience in the use of ECMO as a bridge to lung transplant is likely the result of a small number of highly experienced centers, and the improvement in survival may be a manifestation of improvement in these individual centers rather than nationwide as a whole. Therefore, this selective experience may not necessarily be reproducible at centers with little or no experience in extracorporeal support, and obviously caution should be applied at such centers. Fourth, the use of administrative databases carries with it an inherent risk of bias, and our focus on only those

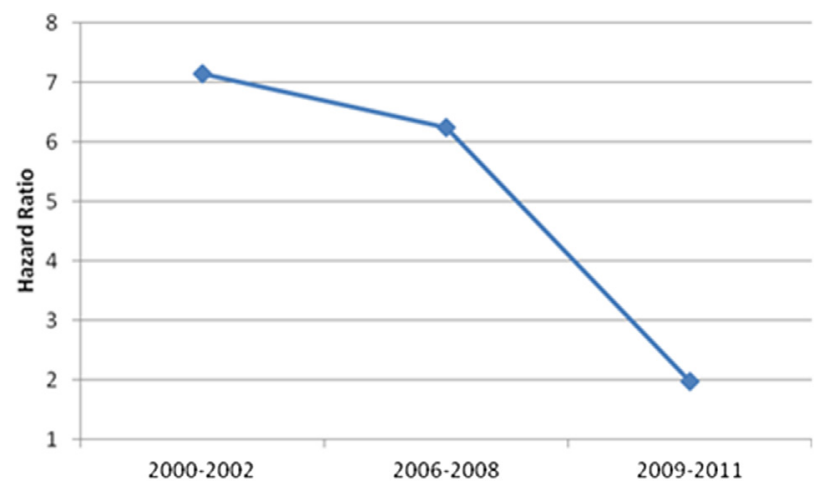

FIGURE 2. Trends in significant HR of 1-year mortality after transplantation for supported with ECMO compared with patients not supported with ECMO. 
TABLE 3. Predictors of 1-year mortality for patients on extracorporeal membrane oxygenation

\begin{tabular}{lcc}
\hline \multicolumn{1}{c}{ Variables } & HR $(\mathbf{9 5} \%$ CI $)$ & $\boldsymbol{P}$ value \\
\hline Age $\geq 35 \mathrm{y}$ & $4.69(1.34-16.46)$ & .02 \\
Cystic fibrosis & $5.38(1.39-20.9)$ & .03 \\
Other* lung diagnoses & $2.49(1.21-5.15)$ & .01 \\
\hline
\end{tabular}

$C I$, Confidence interval; $H R$, hazard ratio. *Other refers to diagnoses other than chronic obstructive pulmonary disease, idiopathic pulmonary fibrosis, or cystic fibrosis.

patients who underwent transplantation (or who were bridged to transplantation) may further underestimate the morbidity and mortality of ECMO bridging without a categorization of the morbidity and mortality of those patients who did not undergo transplantation or who were bridged to an alternative therapeutic option, mechanical or otherwise. This, admittedly, was not the focus of this study. Finally, the study is unable to quantify or adjust for the effect of contemporary improvements in cardiac anesthesia, the transition to the LAS system, echocardiographic diagnostics, blood transfusion protocols, and intensive care delivery, each of which undoubtedly contributed to the overall improvement in survival. The comparison of survival in 2 different eras may be biased in the absence of an ability to adjust for these factors and other generational confounders inherent in the context of constantly evolving medical technology and a dynamic clinical landscape.

\section{CONCLUSIONS}

ECMO is an important strategy in bridging patients with rapidly advancing end-stage pulmonary disease to transplantation. The observed increase in short-term survival in patients bridged to transplantation with ECMO is significant both statistically and clinically, and ultimately provides pertinent information to guide decision making at the bedside when confronted with the question to bridge or not to bridge. The use of a national registry allows the analysis of trends in outcomes using a larger denominator, circumventing the limitations of small center experiences whose analyses have thus far been limited by type 2 error and as such have largely been inconclusive in their recommendations. Thus, on the basis of this study, it is our

TABLE 4. Propensity score-adjusted risk of dialysis-dependent renal failure after transplantation comparing extracorporeal membrane oxygenation with nonextracorporeal membrane oxygenation cases stratified into 3-year intervals

\begin{tabular}{ccc}
\hline Period & OR $(\mathbf{9 5} \% \mathbf{C I})$ & $\boldsymbol{P}$ value \\
\hline $2000-2002$ & $3.32(0.32-34.0)$ & .31 \\
$2003-2005$ & $11.10(2.40-51.40)$ & .002 \\
$2006-2008$ & $13.57(6.44-28.6)$ & $<.001$ \\
$2009-2011$ & $3.31(1.66-6.58)$ & .007 \\
\hline
\end{tabular}

$C I$, Confidence interval; $O R$, odds ratio. recommendation that ECMO may be used as a bridge to lung transplantation with satisfactory survival. Our findings support the notion that well-selected candidates with rapidly advancing end-stage pulmonary disease may be safely bridged until a suitable donor is identified. The survival still remains significantly lower than in patients who did not require ECMO support, but this gap appears to be closing. Further research ideally should focus on identifying the most optimal candidates for this approach, the timing of the intervention, when and when not to offer the bridge, and the optimal duration of support. Optimizing each of these would likely serve, in conjunction with advancing clinical technology, to further improve outcomes and survival with ECMO use in transplantation.

\section{References}

1. Christie JD, Edwards LB, Aurora P, Dobbels F, Kirk R, Rahmel AO, et al. The Registry of the International Society for Heart and Lung Transplantation: Twenty-sixth Official Adult Lung and Heart-Lung Transplantation Report2009. J Heart Lung Transplant. 2009;28:1031-49.

2. Lafarge M, Mordant P, Thabut G, Brouchet L, Falcoz P, Haloun A, et al. Experience of extracorporeal membrane oxygenation as a bridge to lung transplantation in France. J Heart Lung Transplant. 2013;32:905-13.

3. Hämmäinen P, Schersten H, Lemström K, Riise GC, Kukkonen S, Swärd K, et al. Usefulness of extracorporeal membrane oxygenation as a bridge to lung transplantation: a descriptive study. J Heart Lung Transplant. 2011;30:103-7.

4. Haneya A, Philipp A, Mueller T, Lubnow M, Pfeifer M, Zink W, et al. Extracorporeal circulatory systems as a bridge to lung transplantation at remote transplant centers. Ann Thorac Surg. 2011;91:250-5.

5. Santambrogio L, Nosotti M, Palleschi A, Tosi D, Mendogni P, Lissoni A, et al Use of venovenous extracorporeal membrane oxygenation as a bridge to urgent lung transplantation in a case of acute respiratory failure. Transplant Proc. 2009 41:1345-6.

6. Mason DP, Thuita L, Nowicki ER, Murthy SC, Pettersson GB, Blackstone EH. Should lung transplantation be performed for patients on mechanical respiratory support? the US experience. J Thorac Cardiovasc Surg. 2010;139: 765-73.e1.

7. Anile M, Diso D, Russo E, Patella M, Carillo C, Pecoraro Y, et al. Extracorporeal membrane oxygenation as bridge to lung transplantation. Transplant Proc. 2013; 45:2621-3.

8. Olsson KM, Simon A, Strueber M, Hadem J, Wiesner O, Gottlieb J, et al. Extracorporeal membrane oxygenation in nonintubated patients as bridge to lung transplantation. Am J Transplant. 2010;10:2173-8.

9. Garcia JP, Iacono A, Kon ZN, Griffith BP. Ambulatory extracorporeal membrane oxygenation: a new approach for bridge-to-lung transplantation. J Thorac Cardiovasc Surg. 2010;139:e137-9.

10. Veith FJ, Koerner SK. Lung transplantation 1977. World J Surg. 1977;2:177-84.

11. Oto T, Rosenfeldt F, Rowland M, Pick A, Rabinov M, Preovolos A, et al. Extracorporeal membrane oxygenation after lung transplantation: evolving technique improves outcomes. Ann Thorac Surg. 2004;78:1230-5.

12. McCurry KR, Shearon TH, Edwards LB, Chan KM, Sweet SC, Valapour M, et al. Lung transplantation in the United States, 1998-2007. Am J Transplant. 2009;9: 942-58.

13. Glassman LR, Keenan RJ, Fabrizio MC, Sonett JR, Bierman MI, Pham SM, et al Extracorporeal membrane oxygenation as an adjunct treatment for primary graft failure in adult lung transplant recipients. J Thorac Cardiovasc Surg. 1995;110; 723-7.

14. Zenati M, Pham SM, Keenan RJ, Griffith BP. Extracorporeal membrane oxygenation for lung transplant recipients with primary severe donor lung dysfunction. Transpl Int. 1996;9:227-30.

15. Nguyen DQ, Kulick DM, Bolman RM III, Dunitz JM, Hertz MI, Park SJ. Temporary ECMO support following lung and heart-lung transplantation. J Heart Lung Transplant. 2000;19:313-6.

16. Diso D, Venuta F, Anile M, De Giacomo T, Ruberto F, Pugliese F, et al. Extracorporeal circulatory support for lung transplantation: Institutional experience. Transplant Proc. 2010;42:1281-2. 
17. Dellgren G, Schersten H, Kjellman U, Gilljam M, Swärd K, Thylén A, et al. [ECMO can be a bridge to lung transplantation. New method saves life in acute pulmonary failure according to a retrospective study]. Lakartidningen. 2011;108: 1493-7.

18. Gregoric ID, Chandra D, Myers TJ, Scheinin SA, Loyalka P, Kar B. Extracorporeal membrane oxygenation as a bridge to emergency heart-lung transplantation in a patient with idiopathic pulmonary arterial hypertension. J Heart Lung Transplant. 2008;27:466-8.

19. Bermudez CA, Rocha RV, Zaldonis D, Bhama JK, Crespo MM, Shigemura N, et al. Extracorporeal membrane oxygenation as a bridge to lung transplant: midterm outcomes. Ann Thorac Surg. 2011;92:1226-32.

20. Hoopes CW, Kukreja J, Golden J, Davenport DL, Diaz-Guzman E, Zwischenberger JB. Extracorporeal membrane oxygenation as a bridge to pulmonary transplantation. Thorac Cardiovasc Surg. 2013;145:862.

21. Jurmann MJ, Schaefers HJ, Demertzis S, Haverich A, Wahlers T, Borst HG. Emergency lung transplantation after extracorporeal membrane oxygenation. ASAIO J. 1993;39:M448-52.

22. Javidfar J, Brodie D, Iribarne A, Jurado J, LaVelle M, Brenner K, et al. Extracorporeal membrane oxygenation as a bridge to lung transplantation and recovery. $J$ Thorac Cardiovasc Surg. 2012;144:716-21.

23. Bittner HB, Lehmann S, Rastan A, Garbade J, Binner C, Mohr FW, et al. Outcome of extracorporeal membrane oxygenation as a bridge to lung transplantation and graft recovery. Ann Thorac Surg. 2012;94:942-50.

24. George TJ, Beaty CA, Kilic A, Shah PD, Merlo CA, Shah AS. Outcomes and temporal trends among high-risk patients after lung transplantation in the United States. J Heart Lung Transplant. 2012;31:1182-91.

25. Toyoda Y, Bhama JK, Shigemura N, Zaldonis D, Pilewski J, Crespo M, et al. Efficacy of extracorporeal membrane oxygenation as a bridge to lung transplantation. J Thorac Cardiovasc Surg. 2013;145:1065-71.

26. Peek GJ, Killer HM, Reeves R, Sosnowski AW, Firmin RK. Early experience with a polymethyl pentene oxygenator for adult extracorporeal life support. ASAIO J. 2002;48:480-2.

27. Fuehner T, Kuehn C, Hadem J, Wiesner O, Gottlieb J, Tudorache I, et al. Extracorporeal membrane oxygenation in awake patients as bridge to lung transplantation. Am J Respir Crit Care Med. 2012;185:763-8.

28. Nosotti M, Rosso L, Palleschi A, Lissoni A, Crotti S, Marenghi C, et al. Bridge to lung transplantation by venovenous extracorporeal membrane oxygenation: a lesson learned on the first four cases. Transplant Proc. 2010;42:1259-61.

29. Marasco SF, Lukas G, McDonald M, McMillan J, Ihle B. Review of ECMO (extra corporeal membrane oxygenation) support in critically ill adult patients. Heart Lung Circ. 2008;17(Suppl 4):S41-7.

\section{Discussion}

Dr D. Mason (Cleveland, Ohio). Jay, I enjoyed the talk. You have made a good argument and presented good evidence that it is appropriate to use ECMO for well-selected transplant recipients.

I have 3 questions. First, you allude to changes in ECMO technology accounting for the difference between more recent survival and more historic survival outcomes. Do you think this is all due to changes in the actual technology or there might be other, more important factors at play that account for these differences?

Second, are you able from the data to tell us whether these cases are being done at a few centers with larger experiences or are these patients undergoing transplantation at multiple centers with small experiences?

The final question has to do with patient selection for bridging. From the data or your own personal experience, are you able to give some idea to the group about the inclusion/exclusion criteria for patients undergoing transplantation with ECMO?

Dr Bhama. The other factors besides the technology is an important question, and I alluded to that somewhat in the "Study Limitations" section. We didn't account for a number of things that probably play a role in why we have better outcomes today. I would say that some of those things include things such as better donor and recipient selection. I think we make smarter decisions about who undergoes transplantation and which donors we use. There has been a clear impact with the LAS score. Wait times are shorter. Sicker patients are getting organs, probably better now than they were in the past. Other things that may contribute are newer ways of applying ECMO. In the recent era, we didn't look at this, but there is a lot of interest in ambulatory ECMO, applying ECMO in less sick patients. A number of these factors probably play an important role. We were motivated by the improvement in other areas, such as in heart failure and cardiac arrest. We have seen ECMO outcomes improve across the board. We thought this was probably related to the technology, but I think you are right, there are a lot of other factors.

With regard to your second question, we didn't look at the center volume and where these procedures were being done, but it is our suspicion that this experience probably reflects the experience of a couple of centers. That is why we also think it is probably difficult to generalize this to other, maybe less busy centers or centers that don't have as much experience. We would have to look into that.

Regarding your last question in terms of patient selection, at Pittsburgh we tend to be aggressive with regard to the use of ECMO in these patients, and we have a large program. I would say that although we do individualize the strategy on the basis of each patient, we tend to shy away from this strategy in older patients. As we get to the age of 70 years, for instance, we start to think less about ECMO in patients with other comorbid conditions, such as renal failure, frailty, poor nutrition, long intensive care unit stay, long ventilator time, or on ECMO for a long time. We take all of this into account, but it is really individualized.

Dr T. Egan (Chapel Hill, NC). Your early era spanned the introduction of the LAS, and it would be helpful for you to break up that early era. I think what you will find is that there was little ECMO-totransplant before LAS and that the LAS really allowed access to these organs and probably improved survival in that group.

Dr Bhama. I agree with that completely. 